\title{
Síndrome antifosfolípido y necrosis suprarrenal, reporte de dos casos y revisión de la literatura científica
}

\author{
J. Nicolás Fernández Bonilla ${ }^{1}$, Luis Fernando Jaramillo García ${ }^{2}$, Paola Escalante ${ }^{3}$
}

\section{Resumen}

El síndrome antifosfolípido es una entidad que puede ocurrir de forma aislada o asociada a otras enfermedades. Una forma infrecuente es la manifestación con compromiso de las glándulas suprarrenales. Existen pocos casos en la literatura científica que describan este fenómeno. En el presente artículo, se presentan dos casos con comportamientos poco usuales.

Palabras clave: síndrome antifosfolípido, enfermedad de Addison, necrosis.

\section{Title}

Antiphospholipid syndrome and adrenal gland necrosis, report of two cases and review of the literature

\begin{abstract}
Antiphospholipid syndrome is a disease that can manifest alone or associated with other conditions. Adrenal glands involvement is a rare presentation. There are few cases reported in the literature. We present two cases of patients with antiphospholipid syndrome that compromise the adrenal glands and behave very odd comparing to previous case reports.
\end{abstract}

Key words: antiphospholipid syndrome, Addison's disease, necrosis.

1 Médico residente, Departamento de Urología, Pontificia Universidad Javeriana, Bogotá, D.C., Colombia.

2 Médico Patólogo, Director, Departamento de Patología, Hospital San Ignacio, Pontificia Universidad Javeriana. Profesor Facultad de Medicina, Pontificia Universidad Javeriana. Bogotá, D.C., Colombia.

3 Médica, residente, Departamento de Patología, Pontificia Universidad Javeriana, Bogotá, D.C., Colombia. 


\section{Introducción}

La insuficiencia suprarrenal primaria, o enfermedad de Addison, es una entidad que fue descrita por primera vez en 1873. Simultáneamente, Brown Sequard describió, luego de practicar adrenalectomías a ratones de laboratorio, la importancia funcional de la glándula. Es una entidad poco frecuente, con una incidencia de 4,7 a 6,2 por cada millón de pacientes[1].

La principal causa en la actualidad es por enfermedad autoinmunitaria $(80 \%)$, seguida de tuberculosis $(15 \%)$ y otras causas (5\%)[2]. Entre las causas menos frecuentes están los fenómenos isquémicos, relacionándose así con el síndrome antifosfolípido. Esta relación fue descrita por primera vez en 1988. Actualmente, se calcula que, de los pacientes con síndrome antifosfolípido, 0,4\% desarrollan la enfermedad de Addison como complicación[3]. Puede ocurrir de forma aguda y catastrófica, como en el caso de síndrome de Asherson, o, por el contrario, de una forma subaguda o crónica[4]. En algunos reportes de caso, la enfermedad de Addison puede ser la manifestación inicial del síndrome antifosfolípido.

En el presente artículo se describen dos casos de necrosis suprarrenal asociada a síndrome antifosfolípido. Cada uno de ellos es particular en su presentación, ya que uno fue una manifestación bilateral y catastrófica, y el otro fue unilateral y prácticamente asintomático.

\section{Reporte de los casos}

\section{Caso 1}

Se trata de una mujer de 36 años de edad, remitida a nuestra institución por un cuadro clínico de dos meses de evolución de dolor abdominal constante, difuso y de tipo cólico, sin otras alteraciones adicionales. Además, presentaba hematemesis en cuncho de café, por lo cual se le practicaron otros estudios; entre ellos una tomografía computadorizada (TC) de abdomen (figura 1), en el cual se identificó una lesión en la glándula suprarrenal derecha, de 33 por $21 \mathrm{~mm}$, que no presentaba realce con el medio de contraste, de características homogéneas y sin claro compromiso de tejidos anexos.

A su ingreso, la paciente se encontraba en buenas condiciones generales, con signos vitales dentro de límites normales. En el examen cardiopulmonar se observó reforzamiento de $\mathrm{S} 2$, sin otros hallazgos específicos. El abdomen era blando, sin masas ni visceromegalias. Ante estos hallazgos, fue manejada en la institución, descartándose el diagnóstico de feocromocitoma por los exámenes de laboratorio.

Como antecedentes de importancia, tenía un diagnóstico síndrome antifosfolípido por siete abortos previos, una trombosis venosa profunda del miembro inferior izquierdo y tromboembolia pulmonar, manejada con anticoagulación. De forma secundaria a la tromboembolia, había desarrollado hipertensión pulmonar grado IV. El resto de los antecedentes fueron negativos. 


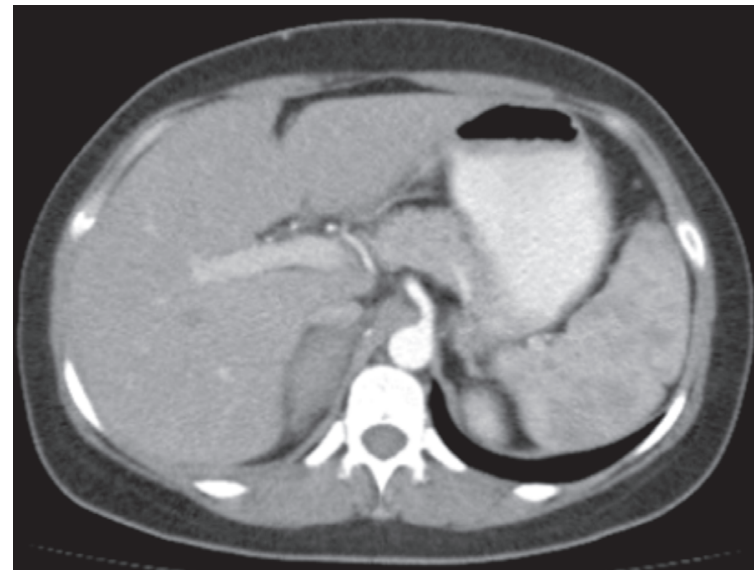

A

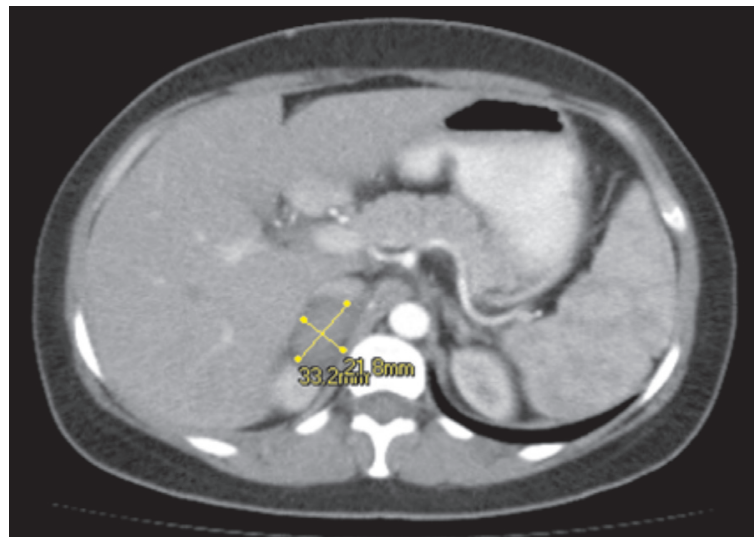

B

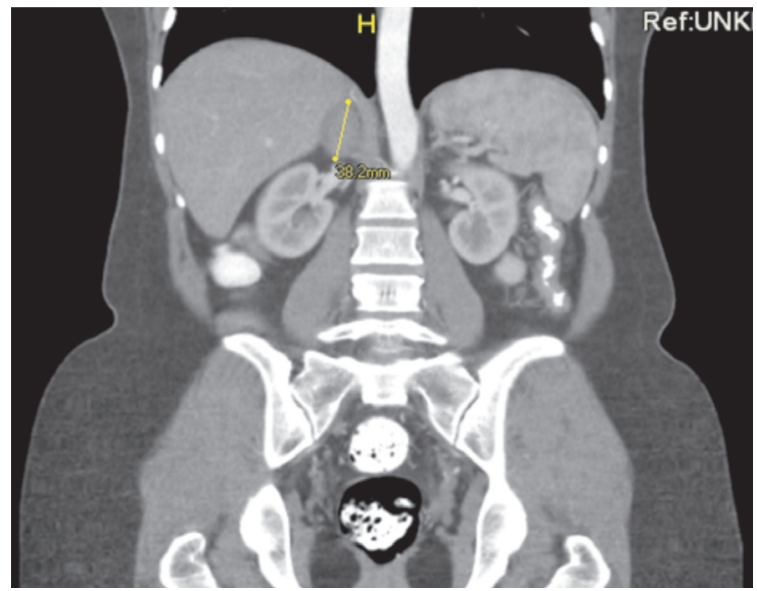

C

Figura 1. Tomografía computadorizada de abdomen. Cortes axiales y coronales en fase arterial (A, B y C). Se identifica una masa suprarrenal derecha de 33 × 21 x 38 mm, hipodensa y homogénea, sin compromiso de tejidos anexos. 
Por los hallazgos de una masa identificada incidentalmente, sin síntomas claros, se decidió practicar adrenalectomía laparoscópica. El procedimiento se llevó a cabo sin complicaciones, con un posoperatorio adecuado.

En el estudio anatomohistólogico de la adrenalectomía se observó tejido con superficie amarillenta y regular al examen macroscópico. Al corte, había importante expansión medular por un tejido rojizo homogéneo. En el examen microscópico, se visualizó una extensa área de necrosis, con escaso tejido suprarrenal. Entre los estudios de inmunohistoquímica, la población celular viable tenía una reacción positiva con citoqueratina y cromogranina. Figura 2 (placas de histología).
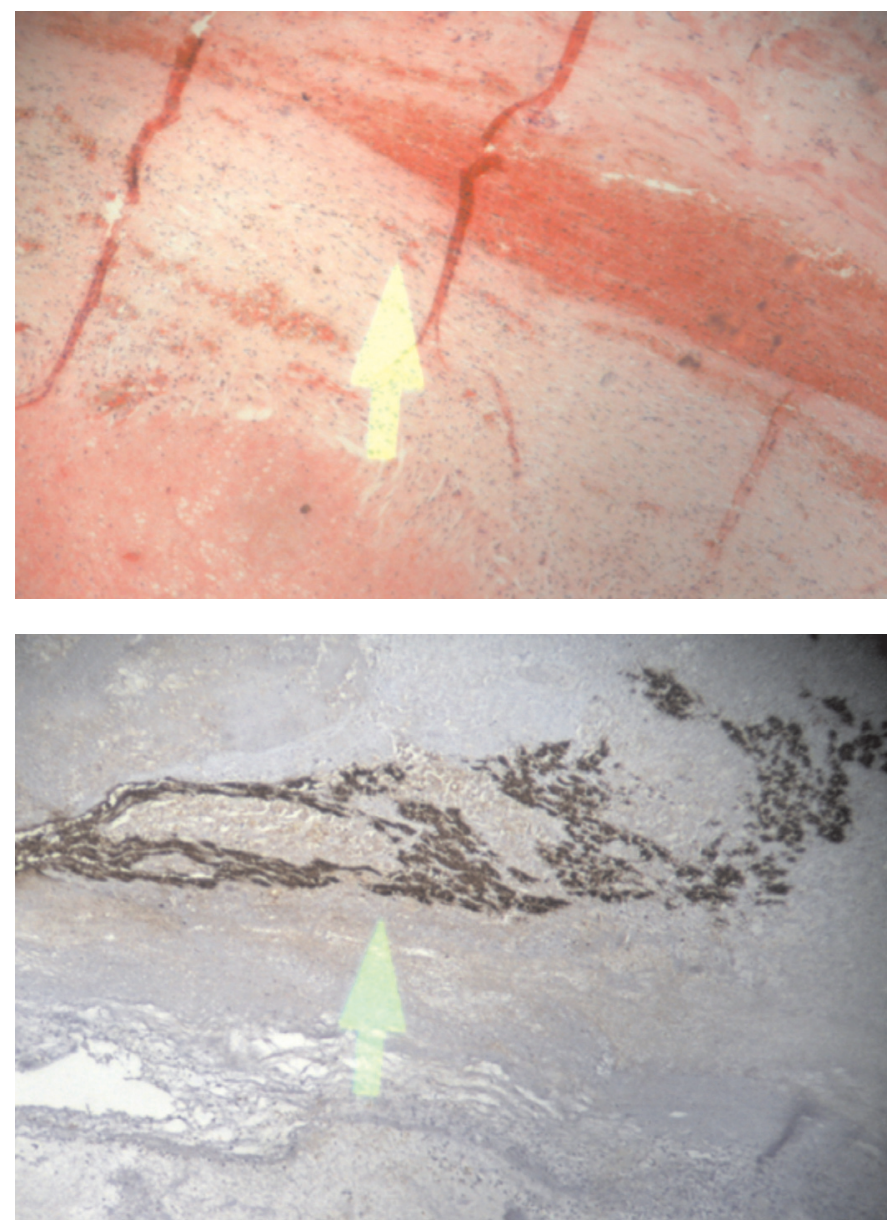

B

Figura 2. Imagen microscópica. A. Se identifican áreas de extensa necrosis. Hematoxilina y eosina, 100x. B. Se identifican escasas células de tejido suprarrenal rodeadas por tejido necrótico. Tinción de inmunohistoquímica con cromogranina, 100x 
Dado el diagnóstico de necrosis suprarrenal unilateral asintomática, se decidió dejar a la paciente sin tratamiento específico y continuar con controles regularmente.

\section{Caso 2}

Se trata de una mujer de 55 años que ingresó al servicio de urgencias por un cuadro clínico de trombosis venosa profunda del miembro inferior izquierdo. Se encontraba en manejo con anticoagulación (5 y 2,5 mg interdiarios de warfarina) con un INR en rango terapéutico por un episodio de trombosis venosa profunda nueve meses antes del cuadro actual. Ante estos hallazgos, se decidió hospitalizarla e iniciar anticoagulación con heparina no fraccionada.

Entre sus antecedentes patológicos, refirió osteoartrosis y, entre los quirúrgicos, histerectomía por miomatosis uterina. Los ginecoobstétricos fueron de dos embarazos con parto y ningún aborto; su última menstruación fue cinco años atrás. Fue fumadora de una cajetilla al día durante diez años.

En la revisión por sistemas, lo único positivo era una pérdida de peso no justificada en los últimos seis meses, asociada a síntomas constitucionales como astenia, adinamia y sudoración nocturna.

Durante la hospitalización, inicialmente presentó estabilidad hemodi- námica. Al quinto día de hospitalización, presentó un episodio súbito de cefalea, para lo cual se practicaron imágenes diagnósticas y se encontró trombosis de los senos venosos. Se continuó con tratamiento de anticoagulación, y presentó deterioro clínico progresivo. Tres días después presentó dolor abdominal agudo, sin signos claros de irritación peritoneal. Se tornó hipotensa, para lo cual fue necesario dar manejo agresivo de reanimación hídrica, sin obtenerse mejoría, razón por la cual se inició tratamiento vasopresor de soporte. La paciente no mejoró con el tratamiento administrado y falleció.

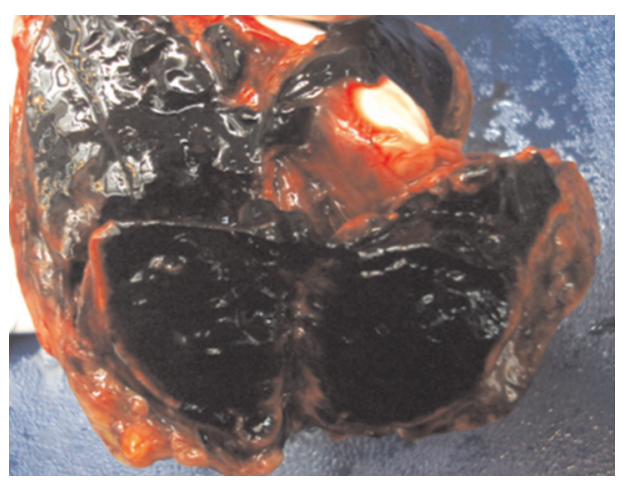

Figura 3 (muestra macroscópica de glándula suprarrenal).

\section{Discusión}

El síndrome antifosfolípido es una entidad autoinmunitaria caracterizada por una propensión a desarrollar trombosis. Fue descrita por primera vez en 1986, es una enfermedad que puede ocurrir en asociación con otras enfermedades autoinmunitarias, como el 
lupus eritematoso sistémico, o también de forma aislada[5]. Entre los criterios diagnósticos, clínicamente es necesario que el paciente desarrolle una trombosis (trombosis venosa profunda, accidente cerebrovascular), presente uno o más abortos no explicados por otras causas, o tenga pruebas positivas de laboratorio. Estas pruebas deben ser positivas en dos oportunidades separadas por seis meses. El síndrome antifosfolípido puede ser primario, o secundario cuando se asocia a otras enfermedades autoinmunitarias o al uso prolongado de clorpromazina[6].

La primera descripción de un caso de insuficiencia suprarrenal y síndrome antifosfolípido, fue hecha en el año de 1989 por Carette et al.[7]. En 2003, Espinosa et al. analizaron 86 casos, en los cuales se estableció la relación entre el síndrome antifosfolípido y la insuficiencia suprarrenal. En esta serie, en más de un tercio de los pacientes, la primera manifestación del síndrome antifosfolípido fue la insuficiencia suprarrenal[8]. La mayoría de los casos reportados correspondía al síndrome de Asherson, en el que había compromiso de múltiples órganos, como en el caso 2 reportado por nosotros.

\section{Manifestación clínica}

En cerca de la tercera parte de los casos hay un factor desencadenante asociado, por ejemplo, una intervención quirúrgica[9], los estados protrombóticos, como el embarazo o las infecciones sistémicas, o la suspensión abrupta del tratamiento anticoagulante. La edad promedio de presentación es de 42 años y se ha descrito un predominio del sexo masculino en $75 \%$ de los casos. En 70\% de los casos reportados, el síndrome antifosfolípido es primario. Entre las manifestaciones más frecuentes, se encuentra el dolor abdominal seguido de fiebre e hipotensión[10, 11]. No resulta infrecuente que no haya manifestaciones clínicas[12]. En 35\% de los casos, la primera manifestación del síndrome antifosfolípido es el compromiso suprarrenal[8].

Por lo general, en las imágenes diagnósticas es característico ver las glándulas afectadas aumentadas de tamaño $y$, según el tiempo de evolución, se visualizará inicialmente más hiperdensa y, posteriormente, isodensa o hipodensa. En el caso 1, por el tiempo de evolución, la masa se veía más hipodensa.

\section{Fisiopatología}

En el síndrome antifosfolípido se forman anticuerpos contra los fosfolípidos de la membrana, unidos a proteínas plasmáticas. Estos son las anticardiolipinas y el anticoagulante lúpico. Este último es más específico, mientras que las anticardiolipinas son más sensibles[13]. El punto crítico de estos anticuerpos es que van dirigidos contra la $\beta 2$-glucoproteína I, asociada al tejido endotelial, la cual al ser activada por la interacción con los anticuerpos va 
a facilitar la coagulación en el endotelio[3].

La irrigación de la glándula suprarrenal está dada por tres arterias principales, las cuales se ramifican en 50 a 60 arteriolas[8]. Se favorece el fenómeno isquémico-hemorrágico, ya que presenta una rica irrigación arterial, pero la transición al drenaje venoso es abrupta y con facilidad sobrepasa la posibilidad de drenaje, causando estasis venosa. Cuando, además, se sobrepone un fenómeno trombótico, la estasis es peor y se produce una hemorragia catastrófica[4].

Otra posibilidad etiológica es aquella en la que los pacientes con síndrome antifosfolípido inician su manejo con anticoagulantes y desarrollan un fenómeno hemorrágico en la glándula. No se conoce la frecuencia de este efecto secundario; sin embargo, es infrecuente y más cuando los niveles de las plaquetas están por encima de un recuento de $50.000 \mathrm{mg} / \mathrm{dl}[14]$.

\section{Manejo}

El tratamiento instaurado depende completamente del diagnóstico oportuno y acertado. En los casos de síndrome antifosfolípido manifestado inicialmente con insuficiencia suprarrenal, el hacer correctamente el diagnóstico es difícil.

Una vez hecho el diagnóstico, en $84 \%$ de los casos, el inicio de la administración de esteroides es el primer paso, seguido de inicio de anticoagulación y antiagregación. En casos más graves, como en el síndrome de Asherson, se pueden considerar tratamientos más agresivos, como la plasmaféresis. Sin embargo, estos son casos en los cuales se requiere un manejo agresivo y multiorgánico en la unidad de cuidados intensivos. No hay datos en la literatura científica sobre la mortalidad de estos pacientes.

\section{Conclusiones}

El compromiso de la glándula suprarrenal en pacientes con síndrome antifosfolípido es infrecuente pero importante de tener en cuenta, ya que puede ser catastrófico y requiere un diagnóstico inmediato. En el presente artículo se presentan los dos extremos de las manifestaciones, con un cuadro clínico muy poco sintomático y de buena evolución, y otro caso mucho más agresivo y letal.

\section{Bibliografía}

1. Arlt W, Allolio B. Adrenal insufficiency. Lancet. 2003;361:1881-93.

2. Betterle C, Dal Pra C, Mantero F, Zanchetta R. Autoimmune adrenal insufficiency and autoimmune polyendocrine syndromes: Autoantibodies, autoantigens, and their applicability in diagnosis and disease prediction. Endocr Rev. 2002;23:327-64.

3. Levine JS, Branch DW, Rauch J. The antiphospholipid syndrome. $N$ Eng $J$ Med. 2002;346:752-63. 
4. Asherson RA, Cervera R, Piette JC, Shoenfeld Y, Espinosa G, Petri MA et al. Catastrophic antiphospholipid syndrome. Clues to the pathogenesis from a series of 80 patients. Medicine (Baltimore). 2001;80:355-77.

5. Fujishima N, Komatsuda A, Ohyagi H, Fujishima M, Tada M, Ohtani $\mathrm{H}$ et al. Adrenal insufficiency complicated with antiphospholipid syndrome. Intern Med. 2006;45:963-6.

6. Böber E, Kovanlikaya A, Büyükgebiz. Primary antiphospholipid síndrome: An unusual cause of adrenal insufficiency. Horm Res. 2001;56:140-4.

7. Carette S, Jobin F. Acute adrenal insufficiency as a manifestation of the anticardiolipin syndrome. Ann Rheum Dis. 1989;48:430-1.

8. Espinosa G, Cervera R, Font J, Asherson RA. Adrenal involvement in the antiphospholipid syndrome. Lupus. 2003;12:569-72.

9. Takebayashi K, Aso Y, Tayama K, Takemura Y, Inukai T. Primary antiphospholipid syndrome associated with acute adrenal failure. Am J Med Sci. 2003;325:41-4.

10. Ishizuka T. Adrenal insufficiency complicated with antiphospholipid syndrome. Intern Med. 2006;45:1077-8.

11. Alfadhli E. Secondary adrenal insufficiency and primary antiphospholipid syndrome. Intern Med. 2009;48:1963-6.

12. Asherson R, Hughes G. Addison's disease and primary antiphosphlipid syndrome. Lancet. 1989;ii:874.

13. Presotto F, Fornasini F, Betterle1 C, Federspil G, Rossato M. Acute adrenal failure as the heralding symptom of primary antiphospholipid syndrome: Report of a case and review of the literature. European Journal of Endocrinology. 2005;153:507-14.

14. Italian Registry of antiphospholipid síndrome (idiopathic and secondary to SLE): first report from the Italian registry. Haematologica. 1993;78:313-8. 